\title{
A Harmony Theory Network solution To The N-Queens Problem
}

T.Tambouratzis, Inst. of Informatics and Telecommunications, NCRPS "Demokritos", Aghia Paraskevi 153 10, Attiki, Greece.

A parallel implementation of the Artificial Intelligence $\mathrm{N}-$ Queens problem is presented. The problem consists of placing the greatest possible number of queens in an NxN chessboard in such a way that no queen can threaten or be threatened by any other queen. It has been proved that the maximum number of mutually non-attacking queens equals $N$. The N-queens problem has traditionally been solved by depth-first search with chronological back-tracking [1]. This however is not very efficient since: a) a state can be proved invalid very late (e.g. while placing the Nth queen) whereas the error is due to the location of the first queen, and b) there exists a very limited number of solutions to the problem (e.g. for $\mathrm{N}=4$, out of the 1820 combinatorially possible states with 4 queens placed on the $4 \times 4$ chessboard, only 2 constitute valid solutions). Recently, more economical search algorithms have been proposed [2].

Here, the $\mathrm{N}$-Queens problem is expressed as a constraintpropagation task. The constraints are dictated by the locations of the NxN chessboard which are invalidated once a queen is placed on the chessboard. Hence, after placing a queen, no other queen is allowed to be located on the same: 1- row, 2-column, 3- right diagonal, and 4- left diagonal of the chessboard (such two queens would mutualiy threaten each other). These constraints can then be propagated in order to find a solution to the N-Queens problem.

A Harmony Theory network [3] is employed to solve the Nqueens problem, since Harmony Theory supports parallel implementation of tasks solved by constraint-propagation. Requiring no training phase, Harmony Theory is especially suited to problems whose regularities (constraints, requirements) are clearly stated. The lower layer of the network consists of $\mathrm{NxN}$ nodes, each node representing the state of one square of the $\mathrm{NxN}$ chessboard (whether it is occupied by a queen or not). The upper layer consists of nodes encoding each of the constraints 1-, 2-, 3- and 4- for every square of the chessboard. Connections are only enforced between the two layers and specify which squares of the chessboard may become simultaneously occupied. Weights are mathematically defined [3].

The presented Harmony Theory network implementation has been found to be able to optimally solve the $\mathrm{N}$-Queens problem by achieving to place $N$ queens in compatible positions. The parallel constraint-propagation formulation of search has furthermore been found to obliterate the need of employing heuristics or back-tracking.

1. Kale, L.V., (1990). "An Almost Perfect Heuristic For The $N$ Nonattacking Queens Problem". Information Processing Letters, $\underline{34}$, pp. 173-178.

2. Sosic, R., Gu, J., (1991). "Fast Search Algorithms For The N-Queens Problem". IEEE-SMC, 21, pp.1572-1576.

3. Smolensky, P., (1986). "Foundations of Harmony Theory", pp. 194-281, in "parallel Distributed Processing : Foundations". 